finds for the coordinates

$$
\begin{aligned}
& t=t_{0}\left(\alpha^{2} C h s-\beta^{2}\right)+r \cos \varphi \cdot \alpha S h s+r \sin \varphi \cdot \alpha \beta(C h s-1) \\
& x=t_{0} \cdot \alpha S h s+r \cos \varphi \cdot C h s+r \sin \varphi \cdot \beta S h s \\
& y=t_{0} \cdot \alpha \beta(1-C h s)-r \cos \varphi \cdot \beta S h s+r \sin \varphi \cdot\left(\alpha^{2}-\beta^{2} C h s\right) \\
& z=
\end{aligned}
$$

One verifies, that $t^{2}-x^{2}-y^{2}-z^{2}=t_{0}^{2}-r^{2}-z_{0}^{2}$, and that for $\beta=0$ the formulae reduce to those of the rectilinear hyperbolic acceleration found above.

Putting $t_{0}=0$ one finds the time tracks of a rigid body occupying the instantaneous present space at the origin of time. This motion has not been described by Herglotz.

Another conception of a rigid body has been put forward in early discussions, in which it was defined as a collection of points, not time tracks, preserving constant distances among them. The vectors connecting pairs of points which show the constant distance according to this conception need not be normal to the time tracks showing the motions of the points.

Any displacement of such a rigid body of the second kind is an example of a complex revolution in $(1+3)$ dimensional time-space, and in general the axis of such a complex revolution consists of a pair of planes, a 2-dimensional plane in space, and a $(1+1)$-dimensional plane in time-space (a separation plane and an inertia plane, to speak with Robb's terminology ${ }^{4}$ ) which are mutually perpendicular. A continued revolution about a separation plane means an accelerated motion. A revolution about an inertia plane is an instantaneous cyclic circular displacement.

Now it is obvious that one can conceive, as companions of the separation planes $U, V, W \cdots$ considered

${ }^{4}$ A. Robb, A Theory of Time and Space (1914); The Absolute Relations of Time and Space (Cambridge University Press, 1941). before, a series of inertia planes $U^{\prime}, V^{\prime}, W^{\prime} \cdots$, each perpendicular to its companion, every pair defined by a parameter $s$ and effectuating an acceleration or rotation $d \psi=f(s) d s$ and $d \chi=g(s) d s$ respectively. The succession of elements $U(s)$ and $U^{\prime}(s)$ would then generate a collection of time tracks or space curves permitting to locate, for every $s$, a collection of points preserving rigid distances. A few examples have been indicated by Herglotz. ${ }^{1}$

One may take a constant separation plane $U(=O Y Z)$ and a constant acceleration plane $(O X T)$ and make the uniform revolutions defined by $\psi=\alpha u$ and $\chi=\kappa u$. One will have the superposition of a rotation and an acceleration along the axis of rotation

$t=x_{0} S h \psi, x=x_{0} C h \psi, y=r \cos \chi, z=r \sin \chi, \psi / \alpha=\chi / \kappa=u$.

This kind of motions has been called by Herglotz the loxodromic group.

Again take a constant separation plane $U(=O Y Z)$ and a translation in it, the latter $(\lambda \psi)$ being the limit of a rotation about an acceleration plane $U^{\prime}$ at an infinite distance. The resulting group of motions is defined by

$$
t=x_{0} S h \psi, \quad x=x_{0} C h \psi, \quad y=y_{0}+\lambda \psi, \quad z=z_{0}
$$

and has been called by Herglotz the hyperbolic group.

Finally, one may take a stationary duration in time as the limit of an acceleration, and put it together with a circular displacement, $\chi=\omega t$. This leads to $t=t, x=x_{0}, y=r \cos \omega t, z=r \sin \omega t$. These circular motions were called by Herglotz the elliptic group. Obviously for $r \omega \rightarrow 1$ the velocity in the time tracks tends to the velocity of light. For larger $r$ one has no longer time tracks, but spatial curves, and so the particles of this rotating rigid of the second kind cannot extend beyond $r=\omega^{-1}$.

\title{
On the Motion of Test Particles in General Relativity
}

\author{
L. INFELd AND A. SCHILD* \\ University of Toronto, Canada
}

\section{INTRODUCTION}

$\mathbf{I}^{\mathrm{N}}$ this paper we give a simple derivation of the geodesic motion of test particles from Einstein's gravitational equations for empty space. The history of this problem is connected with the development of basic physical concepts.

Classical physics is dominated by a characteristic duality of field and matter. In Newton's theory of

\footnotetext{
*Now at Carnegie Institute of Technology, Pittsburgh,
}

gravitation as well as in the Maxwell-Lorentz theory of electromagnetism the physical laws fall naturally into two independent classes. The first class consists of the partial differential equations which (with suitable boundary conditions at infinity) determine the field in terms of the distribution and motion of the matter which "generates" it. The second class consists of the dynamical equations governing the motion of matter under the forces "exerted" by the field. The complete independence of the dynamical laws from the field 
equations is a direct consequence of the linearity of the field equations.

Early in the development of Einstein's general theory. of relativity, it was suspected that the non-linear character of the gravitational equations made unnecessary the separate assumption of a dynamical law, such as the geodesic postulate for test particles. This important problem was approached by two distinct lines of attack, using different concepts of matter.

One line of attack is based on the existence of an energy momentum tensor and on the equation

$$
R_{\mu \nu}-\frac{1}{2} g_{\mu \nu} R=T_{\mu \nu} .
$$

Here matter is represented by local concentrations of the energy momentum tensor $T_{\mu \nu}$. A compact particle, whose macroscopic interaction with other particles is purely gravitational (i.e., an electrically neutral particle), must be pictured as a narrow world tube of timelike direction, $T_{\mu \nu}$ being essentially different from zero in the interior of the tube and zero in the exterior.

A limiting process may be considered in which the world tube shrinks to a world line while at the same time an integral, involving $T_{\mu \nu}$ and representing the mass of the particle, tends to zero. In this limit a world line is obtained, embedded in a continuous gravitational field; this world line represents a test particle. By virtue of (1.01) and the Bianchi identities, the covariant divergence of $T_{\mu \nu}$ must be zero everywhere. It is a simple consequence of these conservation equations that the world line of a test particle, obtained by the limiting process indicated above, is not arbitrary but must be a geodesic of the continuous metric field obtained by the same limiting process. This important argument was developed by Weyl, ${ }^{1}$ Eddington, ${ }^{2}$ Robertson ${ }^{3}$ and others.

The idea of representing matter by a continuous energy momentum tensor goes back, in part, to Mie's ${ }^{4}$ work on field theory. In its post-relativistic form, Mie's program demands the existence of continuous non-gravitational fields which can represent the elementary particles of nature; $T_{\mu \nu}$ must then be regarded as an explicit function of these new fields. However, this procedure is unsatisfactory on two counts. Firstly, there is at present no consistent theory which represents matter by fields such that all the field variables are free of singularities. Secondly, the division of fields into gravitational components and into components with non-gravitational sources is artificial. We should either have a unitary theory of all fields, or else, restricting ourselves to gravitational phenomena only, consider the gravitational equations without any energy momentum tensor.

The preceding remarks lead us to an alternative procedure which avoids the introduction of an energy

\footnotetext{
${ }^{1} \mathrm{H}$. Weyl, Raum-Zeit-Materie (Verlag. Julius Springer, Berlin 1923), fifth edition, $\$ 38$.

2 A. S. Eddington, The Mathematical Theory of Relativity (Cambridge University Press, London, 1923), $\$ 56$.

${ }^{3}$ H. P. Robertson, Proc. Edinb. Math. Soc. 2, 5, 63 (1936).

${ }^{4}$ G. Mie, Ann. d. Physik 37, 511, 39, 1 (1912); 40, 1 (1913).
}

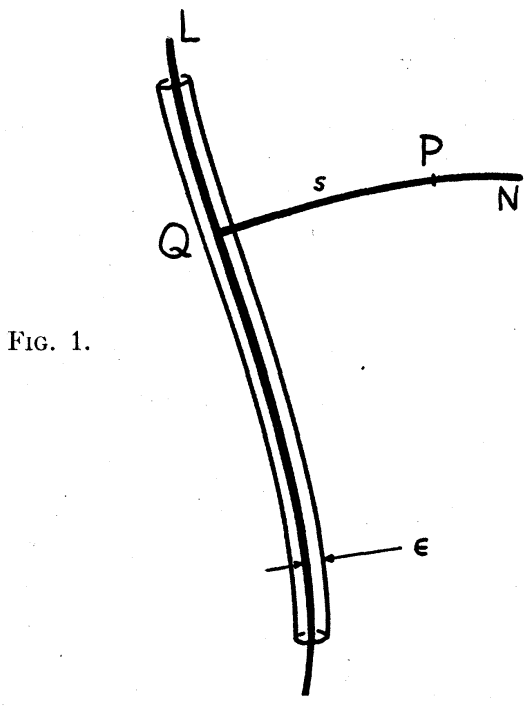

momentum tensor and which is based on a particle picture of matter. Matter is regarded as consisting of point particles; each particle is represented by a singularity of the gravitational field along a time-like world line. The advantage of this model is twofold. Firstly, the gravitational field, satisfying

$$
R_{\mu \nu}-\frac{1}{2} g_{\mu \nu} R=0,
$$

is alone sufficient to represent neutral matter. Secondly, we know (ignoring convergence difficulties) that solutions of (1.02) do exist which represent systems of particles. This was shown by Einstein, Infeld, and Hoffmann ${ }^{5}$. who actually obtained such solutions by an approximation method based on the idea of quasistationary fields. In the same papers it was shown that the motion of the particle singularities is completely determined by the gravitational field equations (1.02) of empty space.

The restricted problem of the geodesic motion of a test particle was attacked ${ }^{6}$ long before the more general $n$-body problem was solved. It might appear that, since the complicated problem of $n$ particles is now solved, the geodesic motion of a test particle should follow as a simple corollary. However, this is not the case, as was drawn to our attention by J. A. Wheeler. The reason is as follows : Einstein, Infeld and Hoffmann ${ }^{5}$ use a new approximation procedure, which is well suited to the case of slowly varying fields, but which is inadequate for our present problem: the motion of a particle of small mass in an arbitrarily strong external field. We shall use a different approximation method proceeding by powers of the mass of the particle.

Let us now ask what exactly we understand by the

${ }^{5}$ A. Einstein, L. Infeld, and B. Hoffmann, Ann. Math. 39, 65 (1938); A. Einstein and L. Infeld, Ann. Math. 41, 455 (1940); a new and improved treatment of the theory will appear shortly in the Canadian J. Math. (1, No. 3).

${ }^{6} \mathrm{~A}$. Einstein and J. Grommer, Sitz Preuss. Akad. Wiss. 1 (1927); K. Lanczos, Zeits. f. Physik 44, 773 (1927). 
geodesic "postulate." A particle is represented by a world line along which the metric field $g_{\mu \nu}$ is singular. We wish to show that this world line is a geodesic in the case of a test particle. Clearly, the statement that a singular line is (or is not) a geodesic has no meaning. Let us however remember that a test particle is defined by a limiting process. Physically, we can consider a sequence of particles, with masses tending to zero, and a corresponding sequence of gravitational fields. In the limit $m=0$ we obtain a limiting world line along which the limiting gravitational field, the background field, is continuous. We must think of the background field as being assigned a prior $i$; the geodesic "postulate" refers to the limiting world line in this continuous field and is thus meaningful. We add a more precise, if less physical, formulation of our problem.

A time-like world line $L_{(0)}$ in a Riemannian 4-space $R_{(0)}$ with coordinates $x^{\rho}$ and metric tensor $g_{(0) \mu \nu}\left(x^{\rho}\right)$, which is analytic at all points of $L_{(0)}$, represents a test particle if the following criterion applies: There exists a sequence of Riemannian 4-spaces $R_{(N)}(N=1,2, \cdots, \infty)$ with coordinates $x^{\rho}$, with a world line $L_{(N)}$ in each, and with a metric tensor $g_{(N) \mu \nu}\left(x^{p}\right)$ which, along $L_{(N)}$, has a singularity of the type representing a particle (see Section 2), such that $\operatorname{Lim}_{N \rightarrow \infty} g_{(N) \mu \nu}\left(x^{\rho}\right)=g_{(0) \mu \nu}\left(x^{\rho}\right)$ for all values of $x^{\rho}$ which in $R_{(0)}$ represent a point not on $L_{(0)}$, and such that all points of $L_{(N)}$ do not tend to infinity as $N \rightarrow \infty$. It follows from this criterion that $\operatorname{Lim}_{N \rightarrow \infty} L_{(N)}=L_{(0)}$; this last relation is to be interpreted by means of the point-point correspondence which exists between the spaces $R_{(N)}, R_{(0)}$ by virtue of their common coordinate system $x^{p}$. The geodesic postulate requires that any $L_{(0)}$ representing a test particle be a geodesic in $R_{(0)}$. This statement, whether true or false, is meaningful, since $g_{(0) \mu \nu}$ is analytic along $L_{(0)}$.

\section{MASS PARTICLE IN GRAVITATIONAL FIELD}

We consider a gravitational field depending on a parameter $m$ (the mass)

$$
g_{\mu \nu}\left(x^{\rho}, m\right)
$$

which is singular on a time-like world line $L$, given by

$$
x^{\rho}=\xi^{\rho}(u) .
$$

We say that this field represents a mass particle moving along the world line $L$ if the following conditions are satisfied:

1. The background field

$$
g_{(0) \mu \nu}\left(x^{\rho}\right)=g_{\mu \nu}\left(x^{\rho}, 0\right)
$$

obtained from (2.01) by putting the parameter $m$ equal to zero, is analytic at every point of $L$, as well as in a neighborhood of $L$.

2. Through a point $P$, not on $L$, there will pass a space-like geodesic $N$ intersecting $L$ orthogonally in a point $Q$ (Fig.1); let $s$ denote the arc length $Q P$. (Here the concepts geodesic, orthogonal, arc length, all refer to the background metric $g_{(0) \mu \nu}$.) Consider the slim tube formed by all points $s=\epsilon$, where $\epsilon$ is a small positive number. Then, for sufficiently small $m$,

$$
m<M(\epsilon),
$$

the metric field (2.01) can be expanded in the form

$$
g_{\mu \nu}\left(x^{\rho}, m\right)=g_{(0) \mu \nu}\left(x^{\rho}\right)+m b_{\mu \nu}\left(x^{\rho}\right)+m^{2} c_{\mu \nu}\left(x^{\rho}\right)+\cdots,
$$

valid at all points outside the tube, such that on the tube, the expressions

$$
\epsilon b_{\mu \nu}, \epsilon^{2} c_{\mu \nu}, \cdots,
$$

remain bounded as $\epsilon \rightarrow 0$ (and, with it, $m \rightarrow 0$ ). Roughly speaking, this condition implies that for a very small mass $m, b_{\mu \nu} \sim 1 / s, c_{\mu \nu} \sim 1 / s^{2}, \cdots$, as the singular world line $L$ is approached.

3. Using the notation of condition 2, we have $g_{\mu \nu} \rightarrow g_{(0) \mu \nu}$ as $s \rightarrow \infty$, or, equivalently, $b_{\mu \nu} \rightarrow 0, \quad c_{\mu \nu} \rightarrow 0$, etc. The gravitational field reduces to the background field at points far removed from the singular world line $L$.

The well-known Schwarzschild solution for a point particle on a flat background is an example of a gravitational field satisfying the three conditions above.

If we put $m=1 / N$, then (2.01) represents a sequence of Riemannian 4-spaces which approach the background field $g_{(0) \mu \nu}$ as $N \rightarrow \infty$ or, equivalently, as $m \rightarrow 0$. It was a sequence such as this which, in the introduction, was used for a precise formulation of the problem of the geodesic motion of a test particle. Thus we wish to show that the gravitational field equations, in the limit $m \rightarrow 0$, impose the condition that the world line (2.02) is a geodesic of the background field.

\section{CHOICE OF COORDINATE SYSTEM}

We choose a coordinate system such that, at all points of $L$,

$$
g_{(0) \mu \nu}=\eta_{\mu \nu}, \quad g_{(0) \mu \nu, \rho}=0 .
$$

Here the comma indicates partial differentiation $\left(g_{(0) \mu \nu, \rho} \equiv \partial g_{(0) \mu \nu} / \partial x^{\rho}\right)$, and $\eta_{\mu \nu}\left(=\eta^{\mu \nu}\right)$ is the Minkowskian metric, given by

$$
\eta_{00}=-\eta_{11}=-\eta_{22}=-\eta_{33}=1, \quad \eta_{\mu \nu}=0 \quad(\mu \neq \nu) .
$$

The existence of such a coordinate system in a general Riemannian space was established by Fermi. ${ }^{7}$

Introducing $x^{0}=t$ as parameter along $L$, (2.02) may be written

$$
x^{r}=\xi^{r}(t) .
$$

Latin suffixes range over 1, 2, 3 while Greek suffixes range over $0,1,2,3$. Putting

$$
z^{r}=x^{r}-\xi^{r}
$$

and expressing $g_{(0) \mu \nu}$ as a function of $z^{1}, z^{2}, z^{3}, t$, we treat the time $t$ as a parameter and consider an expan-

${ }^{7}$ E. Fermi, Rend. Acc. Lincei 21, 21, 51 (1922); T. LeviCivita, Math. Ann, 97, 291 (1927), 
sion of $g_{(0) \mu \nu}$ in powers of $z^{r}$. From (3.01) it follows that

We put

$$
\begin{aligned}
g_{(0) \mu \nu} & =\eta_{\mu \nu}+a_{\mu \nu}, \\
a_{\mu \nu} & =a_{\mu \nu r s}(t) z^{r} z^{s}+\cdots .
\end{aligned}
$$

$$
r=\left(z^{r} z^{r}\right)^{\frac{1}{2}}
$$

and we say that a function $f(z)$ of the $z^{r}$ is at least of order $n$, if $r^{-n} f(z)$ is bounded as $z^{r} \rightarrow 0$. By (3.05), $a_{\mu \nu}$ is of order 2 .

The characteristic properties (3.01) of our coordinate system are clearly invariant under any Lorentz transformation. By special choice of a Lorentz transformation we can, for any assigned point $Q$ of $L$, make

$$
\left(\dot{\xi}^{r}\right)_{Q}=0 .
$$

Here the dot indicates differentiation with respect to the time $t$, and the suffix $Q$ indicates that $\xi^{r}$ is considered at the point $Q$.

\section{THE FIELD EQUATIONS}

The gravitational field equations are

where

$$
G_{\mu \nu} \equiv R_{\mu \nu}-\frac{1}{2} g_{\mu \nu} R=0,
$$

$$
\begin{aligned}
R_{\mu \nu}=-\left\{\begin{array}{c}
\lambda \\
\mu \nu
\end{array}\right\}_{, \lambda}+\left\{\begin{array}{c}
\lambda \\
\mu \lambda
\end{array}\right\}_{, \nu} & \\
& +\left\{\begin{array}{c}
\lambda \\
\mu \sigma
\end{array}\right\}\left\{\begin{array}{c}
\sigma \\
\lambda \nu
\end{array}\right\}-\left\{\begin{array}{c}
\lambda \\
\mu \nu
\end{array}\right\}\left\{\begin{array}{c}
\sigma \\
\lambda \sigma
\end{array}\right\} .
\end{aligned}
$$

Substituting

$$
g_{\mu \nu}=\eta_{\mu \nu}+a_{\mu \nu}+m b_{\mu \nu}+m^{2} c_{\mu \nu}+\cdots,
$$

and separating the different powers of $m$, we find (4.01) becomes

$$
G_{\mu \nu}=A_{\mu \nu}+m B_{\mu \nu}+m^{2} C_{\mu \nu}+\cdots,
$$

where $A_{\mu \nu}$ is the Einstein tensor $G_{\mu \nu}$ of the background field $\eta_{\mu \nu}+a_{\mu \nu}, B_{\mu \nu}$ is linear in $b_{\mu \nu}, C_{\mu \nu}$ is linear in $c_{\mu \nu}$ and quadratic in $b_{\mu \nu}$, etc.

We assume that the background field, which we must regard as assigned $a$ priori, satisfies the gravitational field equations, so that $A_{\mu \nu}=0$. (However, see Section 6 for a generalization.) Also since, in the problem of the geodesic motion of a test particle, we are dealing with the limiting process $m \rightarrow 0$, we ignore all powers of $m$ higher than the first. The field equations then reduce to

$$
B_{\mu \nu}=0 \text {. }
$$

We introduce $\beta_{\mu \nu}$ by the equivalent relations

$$
\beta_{\mu \nu}=b_{\mu \nu}-\frac{1}{2} \eta_{\mu \nu} \eta^{\rho \sigma} b_{\rho \sigma}, \quad b_{\mu \nu}=\beta_{\mu \nu}-\frac{1}{2} \eta_{\mu \nu} \eta^{\rho \sigma} \beta_{\rho \sigma} .
$$

Substituting (4.03) in (4.01), (4.02), we obtain the following expression for $B_{\mu \nu}$ in terms of the $\beta_{\mu \nu}$ :

$$
B_{\mu \nu}=F_{\mu \nu}+L_{\mu \nu}
$$

where

$$
F_{\mu \nu}=\frac{1}{2} \eta^{\rho \sigma}\left\{\beta_{\mu \nu, \rho \sigma}+\eta_{\mu \nu} \eta^{\alpha \beta} \beta_{\alpha \rho, \beta \sigma}-\beta_{\mu \rho, \nu \sigma}-\beta_{\nu \rho, \mu \sigma}\right\},
$$

and $L_{\mu \nu}$ contains products of derivatives of $\beta_{\mu \nu}$ and derivatives of $a_{\mu \nu}$. We write down three typical terms of $L_{\mu \nu}$ :

$$
\eta^{\alpha \beta} \eta^{\rho \sigma} a_{\alpha \rho} \beta_{\mu \beta, \nu \sigma}, \quad \eta^{\alpha \beta} \eta^{\rho \sigma} a_{\mu \beta, \nu \sigma} \beta_{\alpha \rho}, \quad \eta^{\alpha \beta} \eta^{\rho \sigma} a_{\mu \alpha, \rho} \beta_{\nu \beta, \sigma} .
$$

From the discussion of Section 2 it follows that, for a mass particle, $b_{\mu \nu}$, and therefore also $\beta_{\mu \nu}$, are of order -1 in $r$. Since $F_{\mu \nu}$ involves second derivatives of $\beta_{\mu \nu}$, $F_{\mu \nu}$ will contain expressions of order -3 and of higher orders.

Our coordinate system has been chosen such that $a_{\mu \nu}$ is of order 2 in $r$. The typical expressions (4.09) all contain a product of $a_{\mu \nu}$ and $b_{\mu \nu}$, such a product being of order $-1+2=+1$; since each expression in (4.09) involves two differentiations, the lowest order is $+1-2$ $=-1$. Quite generally, it can be seen in this manner that $L_{\mu \nu}$ is of order -1 in $r$.

For small values of $r$, the field equations (4.05) are

$$
F_{\mu \nu}=0
$$

correct to orders -3 and -2 in $r$.

Before discussing any solution of the field equations (4.10), we consider the following substitution ${ }^{8}$ for the variables $\beta_{\mu \nu}$ :

$$
\beta_{\mu \nu}=\bar{\beta}_{\mu \nu}+b_{\mu, \nu}+b_{\nu, \mu}-\eta_{\mu \nu} \eta^{\rho \sigma} b_{\rho, \sigma},
$$

where $b_{\mu}$ are four functions of the space-time coordinates, to be specified shortly. Inserting in (4.08), we find that $F_{\mu \nu}$ is invariant in form under this substitution, i.e.,

$$
F_{\mu \nu}=\frac{1}{2} \eta^{\rho \sigma}\left\{\bar{\beta}_{\mu \nu, \rho \sigma}+\eta_{\mu \nu} \eta^{\alpha \beta} \bar{\beta}_{\alpha \rho, \beta \sigma}-\bar{\beta}_{\mu \rho, \nu \sigma}-\bar{\beta}_{\nu \rho, \mu \sigma}\right\} .
$$

On the other hand

$$
\eta^{\rho \sigma} \beta_{\mu \rho, \sigma}=\eta^{\rho \sigma} \bar{\beta}_{\mu \rho, \sigma}+\eta^{\rho \sigma} b_{\mu, \rho \sigma} .
$$

If we now determine $b_{\mu}$ as a solution of the inhomogeneous wave equation

we have

$$
\eta^{\rho \sigma} b_{\mu, \rho \sigma}=\eta^{\rho \sigma} \beta_{\mu \rho, \sigma}
$$

$$
\eta^{\rho \sigma} \bar{\beta}_{\mu \rho, \sigma}=0 \text {. }
$$

Using this relation it follows that (4.12) simplifies to

$$
F_{\mu \nu}=\frac{1}{2} \eta^{\rho \sigma} \bar{\beta}_{\mu \nu, \rho \sigma} .
$$

Dropping bars (i.e., writing $\beta_{\mu \nu}$ for $\bar{\beta}_{\mu \nu}$ ), one finds that the field equations (4.10) are replaced by

$$
\begin{aligned}
\eta^{\rho \sigma} \beta_{\mu \nu, \rho \sigma} & =0 \\
\eta^{\rho \sigma} \beta_{\mu \rho, \sigma} & =0
\end{aligned}
$$

\footnotetext{
${ }^{8}$ No physical significance need be attached to this change of variables. As indicated in footnote 10, the substitution (4.11) is equivalent to a coordinate transformation to the order required in the present investigation.
} 
We look for a solution $\beta_{\mu \nu}$ of order -1 in $r$, which satisfies Eqs. (4.17b) and which satisfies Eqs. (4.17a) as far as terms of orders -3 and -2 are concerned. As we shall show, such a solution can exist only if $\xi^{r}(t)$ satisfies a certain differential equation-the equation of motion.

Equations (4.17) have a structure similar to Maxwell's equations for the electromagnetic potentials. The only difference is the additional suffix $\mu$ which occurs in the gravitational equations. However, this fact, coupled with the symmetry property $\beta_{\mu \nu}=\beta_{\nu \mu}$ is crucial. Whereas in the electromagnetic case there are 5 differential equations for 4 unknown potentials, there are in the gravitational case 14 equations for only 10 unknowns. This explains why the gravitational field equations determine the motion of singularities, while Maxwell's equations do not determine motion.

In general, a linear field theory cannot determine dynamical equations since an arbitrary external field may be superposed without affecting the world line of a field singularity. However, the linearity of our form (4.17) of the gravitational equations is only apparent. It is a consequence of the special coordinate conditions (3.01) which, for certain orders in $r$, permit us to ignore the non-linear terms $L_{\mu \nu}$. If any external field components $\bar{a}_{\mu \nu}$ were added to the background field $a_{\mu \nu}$, the new background field would no longer satisfy the coordinate conditions and the field equations (4.17) would not apply in the original coordinate system.

\section{EQUATIONS OF MOTION}

With Latin suffixes ranging over 1, 2, 3, we may write the field equations (4.17a) as follows:

$$
\beta_{\mu \nu, m m}-\beta_{\mu \nu, 00}=0 .
$$

We may regard $\beta_{\mu \nu}$ as function of $t$ and of $z^{1}, z^{2}, z^{3}$, defined in (3.04). We shall denote by a dot (as in $\dot{\beta}_{\mu \nu}$ ) a partial derivative with respect to $t$ when $z^{r}$ are kept constant; as before a comma followed by a suffix 0 (as in $\beta_{\mu \nu, 0}$ ) denotes partial differentiation with respect to $t$ when $x^{r}$ are kept constant; a comma followed by a Latin suffix $r$ denotes partial differentiation with respect to either $z^{r}$ or $x^{r}$, the two being equal. Thus, if $f$ is any function of the space-time coordinates, we have

$$
f_{, r}=\partial f / \partial z^{r}=\partial f / \partial x^{r}, \quad f_{, 0}=\dot{f}-f_{, r} \dot{\xi}^{r} .
$$

We can now write (5.01) in the form

$$
\beta_{\mu \nu, m m}+\beta_{\mu \nu, r} \ddot{\xi}^{r}-\ddot{\beta}_{\mu \nu}+2 \dot{\beta}_{\mu \nu, r} \dot{\xi}^{r}-\beta_{\mu \nu, r r} \dot{\xi}^{r} \dot{\xi}^{s}=0 .
$$

We put

$$
\beta_{\mu \nu}=\beta_{-1^{\mu \nu}}+\beta_{0^{\mu \nu}}+\beta_{1^{\mu \nu}}+\cdots,
$$

where $\beta_{-1}^{\mu \nu}$ is the part of $\beta_{\mu \nu}$ which varies as $r^{-1}$, and where $\beta_{0^{\mu \nu}}$ varies as $r^{0}, \beta_{1^{\mu \nu}}$ as $r^{1}$, etc. Note that every differentiation with respect to a $z^{r}$ lowers the order by 1 ; thus $\beta_{-1^{\mu \nu,}, r_{-1}}$ and $\beta_{0^{\mu \nu, m m_{-2}}}$ are both of order -2 . Substituting in (5.03) and equating to zero expressions of order -3 in $r$, and expressions of order -2 , we obtain ${ }^{9}$

$$
\begin{gathered}
\beta_{-1^{\mu \nu, m m_{-2}}}-\beta_{-1^{\mu \nu, r r_{-2}}} \dot{\xi} \dot{\xi}^{s}=0 \\
\beta_{0^{\mu \nu, m m_{-2}}}+\beta_{-1^{\mu \nu, r}, r_{-1} \ddot{\xi}^{r}}+2 \dot{\beta}_{-1}^{\mu \nu, r_{-1}} \dot{\xi}^{r}-\beta_{0^{\mu \nu, r s_{-2}}} \dot{\xi}^{\dot{\xi}} \dot{\xi}^{s}=0
\end{gathered}
$$

Proceeding similarly with $(4.17 \mathrm{~b})$, we obtain the following equations for $\beta_{-1^{\mu \nu}}$ and $\beta_{0^{\mu \nu}}$ :

$$
\begin{array}{r}
\beta_{-1} \mu m, m_{-1} \\
+\beta_{-1}^{\mu 0, r_{-1}} \dot{\xi}^{r}=0, \\
\beta_{0^{\mu m, m_{-1}}}-\dot{\beta}_{-1^{\mu 0}}+\beta_{0^{\mu 0, r_{-1}}} \dot{\xi}^{r}=0 .
\end{array}
$$

These last two equations being valid everywhere except on the singular world line, we may differentiate (5.07) with respect to $t$ keeping $z^{r}$ constant; this gives

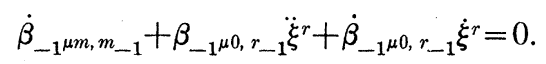

Let us now take any preassigned point $Q$ on the world line $L$ and introduce a coordinate system such that $\dot{\xi}^{r}=0$ for $t=t_{Q}, t_{Q}$ being the time coordinate of $Q$. In the spatial section $t=t_{Q}$, the 5 Eqs. (5.05) to (5.09) simplify to

$$
\begin{aligned}
& \beta_{-1}^{\mu \nu, m m_{-2}}=0, \\
& \beta_{0^{\mu \nu, m m_{-2}}}+\beta_{-1}^{\mu \nu, r} \ddot{\xi}^{r}=0 \text {, } \\
& \beta_{-1^{\mu m, m_{-1}}}=0 \text {, } \\
& \beta_{0^{\mu m, m_{-1}}}-\dot{\beta}_{-1^{\mu 0}}=0 \text {, } \\
& \dot{\beta}_{-1}^{\mu m, m_{-1}}+\beta_{-1^{\mu 0, r_{-1}}} \ddot{\xi}^{r}=0 .
\end{aligned}
$$

From (5.10) we deduce

$$
\beta_{-1}{ }^{\mu \nu}=p_{\mu \nu} / r,
$$

where $p_{\mu \nu}$ are constants (for the fixed time $t_{Q}$ ). Now (5.12) gives

$$
p_{\mu m}=0, \quad \beta_{-1}^{\mu m}=0 .
$$

Putting $\nu=1,2,3$ in (5.11), we have $\beta_{0^{\mu m, n n_{-2}}}=0$; thus $\beta_{0^{\mu m}}$ is a harmonic function, free of singularities and zero at infinity; hence

From (5.13), $\quad \begin{array}{ll}\beta_{0^{\mu m}}=0 . \\ & \dot{\beta}_{-1^{m 0}}=0 .\end{array}$

Finally, putting $\mu=0$ in (5.14), we find

$$
\beta_{-1} 00 ; r_{-1} \ddot{\xi}^{r}=0 .
$$

We come now to an important point of our argument. We wish to show that $p_{00}$ in (5.15) is different from zero, so that (5.19) will give the equation of motion $\ddot{\xi}^{r}$ $=0$. Were it not for the substitution (4.11), the conclusion would be immediate that $\beta_{-1^{00}}$ and therefore also $p_{00}$ must be non-zero; this is so because otherwise, by

${ }^{9}$ Equations (5.01) or (5.03) do not apply to terms of orders higher than -2 in $r$. 
(5.16), all the $\beta_{-1^{\mu \nu}}$ would vanish, the world line $z^{r}=0$ would no longer be a singularity of the $\beta_{\mu \nu}$ field and there would be no mass particle, which is contrary to hypothesis. Because of the substitution (4.11), the argument is less direct. Let us remember that the $\beta_{\mu \nu}$ of this section are the $\bar{\beta}_{\mu \nu}$ of the previous section; Eq. (4.11) shows that the $\beta_{\mu \nu}$ of Section 4 can be singular even if the $\bar{\beta}_{\mu \nu}$ are not. However, it can be shown that, to the order in $r$ required by us, the substitution (4.11) is equivalent to a coordinate transformation. ${ }^{10}$ Thus, when $\bar{\beta}_{\mu \nu}$, are non-singular, no singularity of $\beta_{\mu \nu}$ can represent a mass particle or have physical significance, since it can be wiped out by a coordinate transformation. We conclude that $p_{00} \neq 0$. Summarizing, we can say that the field $\beta_{\mu \nu}$ has the following form in the immediate neighborhood of the singularity:

$$
\beta_{00}=-\frac{4}{r}, \quad \beta_{m 0}=\beta_{m n}=0 .
$$

The numerical value of $p_{00}$ has been chosen such that the parameter $m$ in (4.03) can be identified with the gravitational mass of our particle (when the gravitational constant is taken as 1); this can be seen by comparison with Newtonian gravitational theory or with the Schwarzschild line element.

Equations (5.20) and (5.19) give

$$
\ddot{\xi}^{r}=0 .
$$

This is the required equation of motion. We have proved it of course only for the point $Q$. Remembering the coordinate conditions (3.01) and (3.07), we can write (5.21) in the form

$$
\frac{\delta}{\delta s_{(0)}} \frac{d \xi^{\mu}}{d s_{(0)}} \equiv \frac{d^{2} \xi^{\mu}}{d s_{(0)}^{2}}+\left\{\begin{array}{l}
\mu \\
\rho \sigma
\end{array}\right\} \frac{d \xi^{\rho}}{d s_{(0)}} \frac{d \xi^{\sigma}}{d s_{(0)}}=0
$$

${ }^{10}$ Consider (i) $\bar{x}^{\rho}=x^{\rho}+m \eta^{\rho \sigma} b_{\sigma}$. Under such a transformation which involves $m, g_{\mu \nu}$ transforms tensorially, but $\eta_{\mu \nu}+a_{\mu \nu}$ and $b_{\mu \nu}$ do not. We have

$$
\bar{g}_{\mu \nu}\left(\bar{x}^{\rho}\right)=\frac{\partial x^{\alpha}}{\partial \bar{x}^{\mu}} \frac{\partial x^{\beta}}{\partial \bar{x}^{\nu}} g_{\alpha \beta}\left(\bar{x}^{\rho}-m \eta^{\rho \sigma} b_{\sigma}\right)
$$

Expanding in powers of $m$, we find (ii) $\bar{a}_{\mu \nu}\left(\bar{x}^{\rho}\right)=a_{\mu \nu}\left(\bar{x}^{\rho}\right)$, i.e., $\bar{a}_{\mu \nu}$ are the same functions of $\bar{x}^{\rho}$ as $a_{\mu \nu}$ are of $x^{\rho}$; also

(iii) $b_{\mu \nu}=\bar{b}_{\mu \nu}+b_{\mu, \nu}+b_{\nu, \mu}+\eta^{\rho \sigma}\left(a_{\rho \nu} b_{\sigma, \mu}+a_{\rho \mu} b_{\sigma, \nu}+a_{\mu \nu, \rho} b_{\sigma}\right)$,

(iv) $\beta_{\mu \nu}=\bar{\beta}_{\mu \nu}+b_{\mu, \nu}+b_{\nu, \mu}-\eta_{\mu \nu} \eta^{\rho \sigma} b_{\rho, \sigma}+\lambda_{\mu \nu}$,

where $\lambda_{\mu \nu}$ contains terms of the form $a_{\alpha \beta} b_{\rho, \sigma}$. Because of (ii), the coordinate conditions (3.01) are not affected by (i) except for small terms of order $m$ (the conditions (3.01) apply rigorously to a world line shifted from the singular world line through distances of order $m$ ). In (iv) $\lambda_{\mu \nu}$ is of higher order in $r$ than the remaining terms and it can be ignored if (iv) is applied only to $\beta_{-1} \mu \nu$ and $\beta_{0 \mu \nu}$; then (iv) reduces to (4.11). the suffix (0) indicating that the corresponding quantities refer to the background field. This equation, being tensorial, holds in an arbitrary coordinate system, and $Q$ being a general point on the singular world line $L$, (5.22) holds all along $L$. This establishes our theorem: As a consequence of the gravitational field equations in empty space, a test particle must move along a geodesic of the background field.

We mention briefly an alternative method for obtaining the geodesic postulate. Applying the approximation method of Einstein, Infeld and Hoffmann ${ }^{5}$ to the field equations (4.17), it is easy to obtain a solution in the form of an infinite series. ${ }^{11}$ Subjecting this solution to certain surface integral conditions,,$^{12}$ which follow identically from the field equations (4.17), the result (5.21) is obtained.

\section{GENERALIZATIONS}

Our deduction of the geodesic motion of a test particle remains unaffected if we admit an energy momentum tensor $T_{\mu \nu}$ to the field equations, $T_{\mu \nu}$ being continuous along the world line $L$ of the singularity. The field equations are now (1.01) instead of (1.02). It is clear that the addition of such a $T_{\mu \nu}$ merely changes $L_{\mu \nu}$ in (4.07) by expressions of order 0 in $r$, and it does not affect the terms $F_{\mu \nu}$ of order -3 and -2 , on which the deduction of the geodesic postulate was based.

Physically this means that the geodesic postulate holds for a mass particle in a non-gravitational field (such as an electromagnetic field) if the only interaction between particle and field is gravitational (neutral particle); in this case the field (and thus its energy momentum tensor) have no singularity along the world line of the particle and the above remarks apply.

We may also consider the case when the energy momentum tensor $T_{\mu \nu}$ is singular along the world line $L$. In general, the motion is then no longer along a geodesic. The most important example of such a system is a point charge in an electromagnetic field. This problem was investigated, from the point of view adopted in this paper by Einstein, ${ }^{13}$ and by Infeld and Wallace ${ }^{11}$ who also considered electromagnetic radiation reaction terms but only weak gravitational fields.

${ }^{11}$ See L. Infeld and P. R. Wallace, Phys. Rev. 57, 797 (1940), Eqs. II2.

${ }_{12}$ Reference 11, Eqs. (4.12) with $T_{\mu \nu}=0$.

${ }^{13}$ A. Einstein, Sitz. Preuss. Akad. Wiss., 235 (1927). 